\title{
GÜÇ BILESSENLERINİN DALGACIK DÖNÜŞÜMÜ TABANLI HESAPLANMASI
}

Öz: Sistemler için birçok güç tanımlamaları yapılmıştır. Farklı güç tanımlamaları ve bileşenlerinin doğru bir şekilde hesaplanması ve ölçülmesi son derece önemlidir. Bu amaçla çok sayıda yöntemler ve teknikler geliştirilmiştir. Gerçekleştirilen çalışmada; literatürde tanımlanan gelen-yansıyan-iletilen güç bileşenlerinin geleneksel Fourier dönüşümüne alternatif olarak dalgacık paket dönüşümü kullanılarak hesaplanması önerilmiştir. İlgili güç analizlerini yapmak için etkileşimli grafiksel arayüz programı tasarlanmış; önerilen hesaplama tekniğinin etkinliği ve doğruluğu, gerçekleştirilen benzetimlerle/uygulamalarla gösterilmiştir.

Anahtar Kelimeler: Gelen güç, yansıyan güç, iletilen güç, yansıyan güç kayıp faktörü, dalgacık paket dönüşümü.

\section{The Power Components Calculation based on Wavelet Transform}

\begin{abstract}
Many power definitions have been made for the systems. It is extremely important that different power definitions and their components are accurately calculated and measured. Various methods and techniques have been developed for this purpose. In realized study, it was proposed that calculation of incident-reflected-transmitted power components which are defined in literature can be carried out with wavelet packet transform as an alternative to the traditional Fourier transform. An interactive graphical user interface program was designed to perform related power analysis and the effectiveness and accuracy of the proposed calculation technique was demonstrated by the performed simulations/applications.
\end{abstract}

Keywords: Incident power, reflected power, transmitted power, reflected power loss factor, wavelet packet transform.

\section{GÍRİs}

Modern enerji sistemlerinde, "güç kalitesi” geniş yer tutmaktadır. IEEE'nin Standart IEEE1100’a göre güç kalitesi; cihazlar için uygun güç sağlama ve topraklama koşullarını içeren genel bir kavram olarak tanımlanmaktadır (Şekil 1) (Sankaran, 2002). Güç kalitesini nicelendirmek için de güç büyüklüklerinin ölçülmesi/hesaplanması gerekmektedir.

*Bursa Uludağ Üniversitesi, Mühendislik Fakültesi, Elektrik-Elektronik Müh. bölümü, 16059 Bursa/Türkiye İletişim yazarı: Fahri Vatansever (fahriv@uludag.edu.tr) 


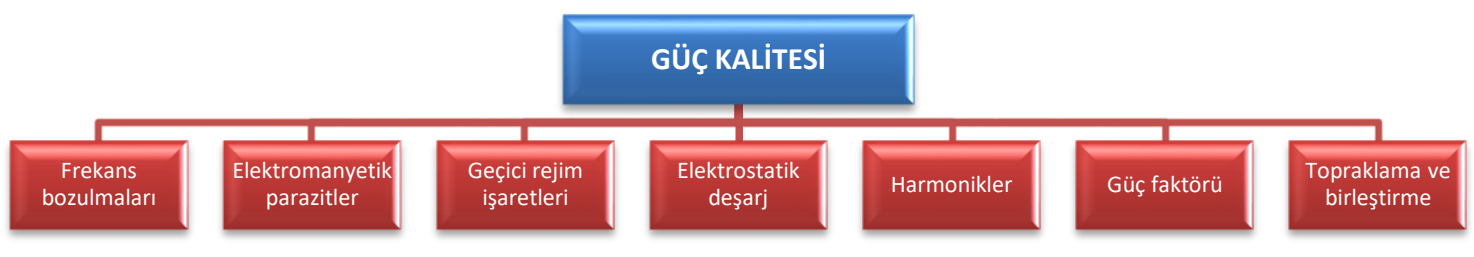

Şekil 1:

Güç kalitesinin ilgili olduğu alanlar

Literatürde değişik koşullar altında farklı güç tanımlamaları yapılmıştır (Budeanu, 1927; Fryze, 1931; Shepherd and Zakikhani, 1972; Sharon, 1973; Kusters and Moore, 1980; Czarnecki, 1985; IEEE, 1988; Slonim and Van der Wyk, 1988; Emanuel, 1990; Filipski, Baghzouz and Cox, 1994; Arseneau et al., 1996). Farklı matematiksel dönüşümler ve teknikler/algoritmalar kullanılarak bu tanımlanan güçlerin hesaplanmaları ve ölçümleri gerçekleştirilmektedir. Geleneksel olarak bu hesaplamalar ve ölçümler çoğunlukla Fourier dönüşümü tabanlı gerçekleștirilmektedir. Simetrik trigonometrik baz fonksiyonları ve sabit pencereleme fonksiyonları kullanan Fourier dönüşümleri ile sürekli rejimdeki statik işaretlerin analizi çok yüksek doğruluklarla gerçekleştirilmektedir. Ancak dinamik işaretlerin analizinde - özellikle geçici rejimlerin veya dinamik işaretlerin eşzamanlı analizinde - problemlerle karşılaşılmaktadır. Dinamik işaretlerin eşzamanlı analizinde, asimetrik baz ve değişken pencereleme fonksiyonları kullanan dalgacık dönüşümleri; işaretleri frekans bantlarına ayrıştırarak ayrı ayrı inceleme imkânı sağlamakta, işarete ait ilgili kısımların yüksek çözünürlükle betimlemesini gerçekleştirebilmekte, sürekli ve geçici rejimleri eşzamanlı analiz edebilmekte, karmaşı matematiksel işlemler (trigonometrik, üstel vb.) gerektirmemekte, sadece sayısal konvolüsyon, veri azaltma gibi basit işlemler kullanmakta (dolayısıyla sayısal sistemlerle yapılmasında basitlik, kolaylık vb. sunmakta) ve temelde çoklu çözünürlük analizine dayanmaktadır (Çankaya ve Vatansever, 2002; Vatansever ve diğ., 2009; Vatansever and Ozdemir, 2010). Bu nedenlerle son yıllarda literatürde ve uygulamalarda dalgacık dönüşümü tabanlı harmonik ve güç yaklaşımları da yer almaktadır (Yoon and Devaney, 1998; Yoon and Devaney, 2000; Hamid, Mardiana and Kawasaki, 2002; Vatansever and Ozdemir, 2008; Vatansever and Ozdemir, 2009; Vatansever and Ozdemir, 2010).

Bu çalışmada; (Lu, Lin and Huang, 2000)'de tanımlanan ve hızlı Fourier dönüşüm algoritmalarıyla hesaplanan güç bileşenlerinin (gelen-yansıyan-iletilen aktif, reaktif ve distorsiyon) ayrık dalgacık paket dönüşümüyle elde edilmesi önerilmektedir. Bu doğrultuda tanımlanan güç bileşenlerine ilişkin dalgacık paket tabanlı eşitlikler sunulmuş ve tasarlanan arayüz programıyla değişik benzetimler/uygulamalar gerçekleştirilmiştir.

\section{DALGACIK PAKET DÖNÜŞÜMÜ}

Herhangi bir $x(t)$ fonksiyonunun sürekli dalgacık dönüşümü (CWT) matematiksel olarak

$$
\left.\begin{array}{l}
W(a, b)=\frac{1}{\sqrt{a}} \int_{-\infty}^{\infty} x(t) \psi\left(\overline{\left.\frac{t-b}{a}\right)} d t\right. \\
x(t)=\frac{1}{C_{\psi}} \int_{-\infty}^{\infty} \int_{-\infty}^{\infty} \frac{1}{a^{2}} W(a, b) \psi_{a, b}(t) d a d b
\end{array}\right\}
$$

denklem çifti ile verilmektedir. Bu eşitliklerde $a>0, b \in \mathfrak{R}$ olmak üzere $a$, ölçekleme/yayılım parametresini; $b$ dönüşüm/öteleme parametresini; $x(t)$, işareti/fonksiyonu; $\psi$, dalgacık fonksiyonunu (ana dalgacığı); $W(a, b)$ da işaretin sürekli dalgacık dönüşümünü ve $C_{\psi}$ da seçilen 
dalgacık türüne bağımlı dalgacık sabitini belirtmektedir. $j, k \in Z$ uygun seçilerek ayrık dalgacık dönüşümü (DWT) de

$$
a=\frac{1}{2^{j}} \quad, \quad b=\frac{k}{2^{j}} \Rightarrow w_{j, k}=W_{\psi} x\left(\frac{k}{2^{j}}, \frac{1}{2^{j}}\right)=\sqrt{2^{j}} \int_{-\infty}^{\infty} x(t) \psi \overline{\left(\frac{t-k / 2^{j}}{1 / 2^{j}}\right)}
$$

eşitliğiyle verilmektedir (Goswami and Chan, 1999; Debnath, 2002). Ayrık dalgacık paket dönüşümünde (DWPT) ise $g[n]$ ve $h[n] ; 2 N$ uzunluğunda, sırasıyla alçak ile yüksek geçiren ve dalgacığa uygun filtreler olmak üzere

$$
\left.\begin{array}{c}
W_{2 n}(t)=\sqrt{2} \sum_{k=0}^{2 N-1} g[k] W_{n}(2 t-k) \\
W_{2 n+1}(t)=\sqrt{2} \sum_{k=0}^{2 N-1} h[k] W_{n}(2 t-k)
\end{array}\right\}
$$

iterasyon eşitlikleri mevcuttur. Eşitlik 3 'te $n=0$ durumunda $W_{0}(t)=\varphi(t)$ ölçekleme fonksiyonu ve $W_{1}(t)=\psi(t)$ de dalgac1k fonksiyonudur (Wickerhauser, 1994; Goswami and Chan, 1999; Debnath, 2002; Mathworks, 2019). Şekil 2'de ayrık $x[n]$ işaretine/fonksiyonuna ait 2 seviyeli DWT ve DWPT ayrışım ağaçları ve bu ayrışımlar sonucu oluşan frekans bantları karşılaştırmalı olarak verilmektedir.

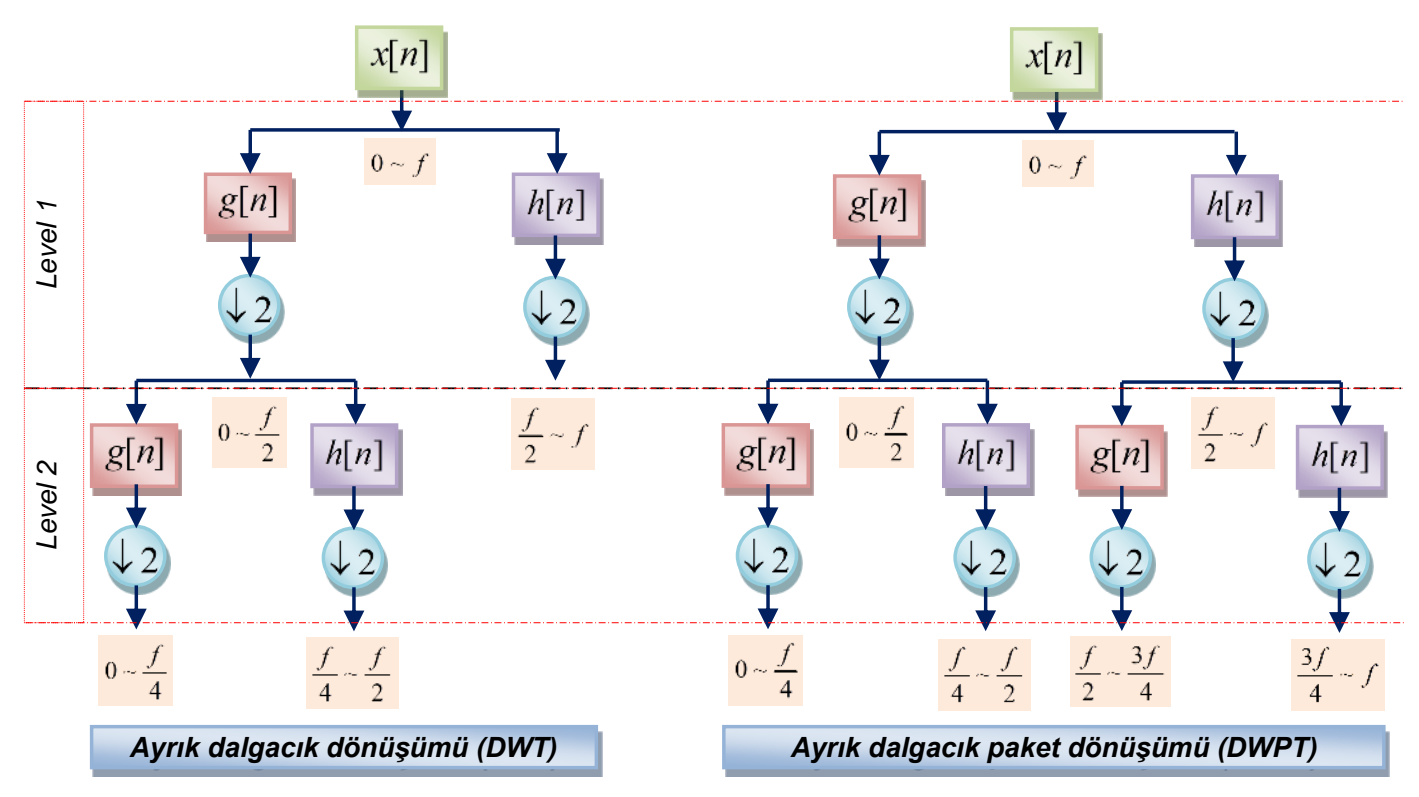

Sekil 2:

2 seviyeli DWT ile DWPT ve frekans bantlart

$N=2^{L}$ örnekten oluşan $x[n]$ ayrık işareti; $s$. seviyeye kadar dalgacık paket ayrışımına tabi tutulursa $2^{s}$ tane düğüm/paket veya frekans bandı oluşmakta ve her bir seviyedeki bantta da $2^{L-s}$ veya $N / 2^{s}$ tane dalgacık paket katsayısı mevcuttur. 2 ile veri azaltmadan dolayı $j$. seviyedeki 
dalgacık katsayılarının sayısı, $(j-1)$. seviyedeki dalgacık katsayılarının sayısının yarısıdır. $s=0,1, \ldots, L$ için $s$. seviye, $m=0,1, \ldots, 2^{s-1}-1$ için $2 m$. düğüm ve $k$. noktadaki dalgacık paket katsayıları $p_{s}^{2 m}[k]$ ile gösterilirse bunlardan $x(t)$ işaretini sentezlemek için

$$
\begin{aligned}
x(t) & =\sum_{m=0}^{2^{s-1}-1} \sum_{k=1}^{N / 2^{s}} p_{s}^{2 m}[k] \psi_{s, k}^{2 m}(t)+\sum_{m=0}^{2^{s-1}-1} \sum_{k=1}^{N / 2^{s}} p_{s}^{2 m+1}[k] \psi_{s, k}^{2 m+1}(t) \\
& =\sum_{k=1}^{N / 2^{s}} p_{s}^{0}[k] \varphi_{s, k}(t)+\sum_{m=1}^{2^{s}-1} \sum_{k=1}^{N / 2^{s}} p_{s}^{m}[k] \psi_{s, k}^{m}(t)
\end{aligned}
$$

eşitliği kullanılmaktadır. Bu eşitlikte $p_{s}^{m}[k], x[n]$ nin dalgacık paket katsayıları ve $p_{s}^{0}[k]$ da ölçekleme fonksiyonu katsayıları veya 0 . düğümdeki dalgacık paket katsayılarıdır (Wickerhauser, 1994; Hamid, Mardiana and Kawasaki, 2002).

\section{GÜÇ ANALIZí}

Şekil 3’teki gibi sinüsoidal gerilim tarafından beslenen doğrusal olmayan yük için (Lu, Lin and Huang, 2000)'de yeni güç tanımlamaları önerilmiş ve bunların hesaplamaları hızlı Fourier dönüşümüyle gerçekleştirilmiştir. Şekil 3' deki yük uçları gerilimi veya iletilen gerilim $v_{t}(t)$, iki bileşene ayrılmaktadır. Bunlar $v_{i}(t)$ gelen gerilim ve $v_{r}(t)$ yansıyan gerilim olup

$$
\left.\begin{array}{l}
v_{i}(t)=\sqrt{2} V_{1} \operatorname{Sin}\left(\omega_{0} t+\alpha_{1}\right) \\
v_{r}(t)=\sqrt{2} \sum_{k=2}^{\infty} V_{k} \operatorname{Sin}\left(k \omega_{0} t+\alpha_{k}\right)
\end{array}\right\} \Rightarrow v_{t}(t)=v_{i}(t)+v_{r}(t)
$$

şeklinde ifade edilmektedirler. Aynı şekilde yük akımı veya iletilen akım $i_{t}(t)$ de iki bileşene ayrılmaktadır: $i_{i}(t)$ gelen akım ve $i_{r}(t)$ yansıyan akım.

$$
\left.\begin{array}{l}
i_{i}(t)=\sqrt{2} I_{1} \operatorname{Sin}\left(\omega_{0} t+\beta_{1}\right) \\
i_{r}(t)=\sqrt{2} \sum_{k=2}^{\infty} I_{k} \operatorname{Sin}\left(k \omega_{0} t+\beta_{k}\right)
\end{array}\right\} \Rightarrow i_{t}(t)=i_{i}(t)+i_{r}(t)
$$

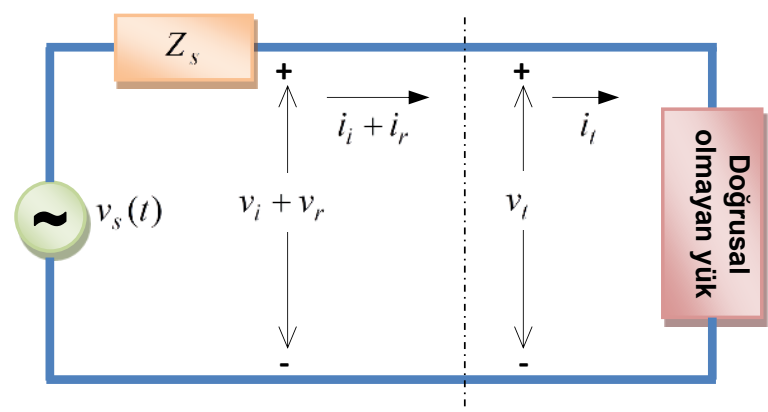

Şekil 3:

Doğrusal olmayan yükü besleyen sinüsoidal kaynaklı devre 
Şekil 3'deki devreye ilişkin (Lu, Lin and Huang, 2000)'de tanımlanan güç bileşenleri, aşağıdaki gibi hesaplanmaktadır.

i. Aktif güç: Doğrusal olmayan yüke iletilen aktif güç $P_{t}$

$$
P_{t}=\sum_{k=1}^{\infty} V_{k} I_{k} \operatorname{Cos}\left(\phi_{k}\right)=\underbrace{V_{1} I_{1} \operatorname{Cos}\left(\alpha_{1}-\beta_{1}\right)}_{P_{i}}+\underbrace{\sum_{k=2}^{\infty} V_{k} I_{k} \operatorname{Cos}\left(\alpha_{k}-\beta_{k}\right)}_{P_{r}}
$$

ile hesaplanmaktadır. Bu eşitlikte gelen aktif güç $P_{i}$, pozitif işaretli ve doğrusal olmayan yük uçlarındaki aktif gücü; yansıyan aktif güç $P_{r}$ ise negatif işaretli olup geriye doğru sisteme yansıyan aktif gücü belirtmektedir. DWPT kullanılarak her bir harmoniğin etkin değeri ve aktif gücünün hesaplanması (Hamid, Mardiana and Kawasaki, 2002)'de yer almaktadır. Bunlar önerilen aktif güç bileşenlerine uyarlanırsa

$$
\begin{gathered}
P_{i}=\frac{1}{N} \sum_{k=1}^{N / 2^{s}} p_{s}^{0, v}[k] p_{s}^{0, i}[k] \\
P_{r}=\sum_{m=1}^{2^{s}-1}\left\{\frac{1}{N} \sum_{k=1}^{N / 2^{s}} p_{s}^{m, v}[k] p_{s}^{m, i}[k]\right\}
\end{gathered}
$$

eşitlikleri elde edilmektedir.

ii. Reaktif güç: Doğrusal olmayan yüke iletilen reaktif güç $Q_{t}$ as

$$
Q_{t}=\sum_{k=1}^{\infty} V_{k} I_{k} \operatorname{Sin}\left(\phi_{k}\right)=\underbrace{V_{1} I_{1} \operatorname{Sin}\left(\alpha_{1}-\beta_{1}\right)}_{Q_{i}}+\underbrace{\sum_{k=2}^{\infty} V_{k} I_{k} \operatorname{Sin}\left(\alpha_{k}-\beta_{k}\right)}_{Q_{r}}
$$

$\mathrm{Bu}$ eşitlikte gelen reaktif güç $Q_{i}$, pozitif işaretli ve doğrusal olmayan yükün uçlarındaki reaktif gücü; yansıyan reaktif güç $Q_{r}$ ise negatif işaretli olup dağıtım sistemine geri yansıtılan harmonik reaktif gücü belirtmektedir. DWPT ve Hilbert dönüşümü kullanılarak reaktif gücün hesaplanması (Vatansever and Ozdemir, 2009)'de yer almaktadır. Bunlar, önerilen reaktif güç bileşenlerine uyarlanırsa

$$
\begin{gathered}
Q_{i}=\frac{1}{N} \sum_{k=1}^{N / 2^{s}} p_{s}^{0, v}[k] p_{s}^{0, \mathcal{H}\{i\}}[k] \\
Q_{r}=\sum_{m=1}^{2^{s}-1}\left\{\frac{1}{N} \sum_{k=1}^{N / 2^{s}} p_{s}^{m, v}[k] p_{s}^{m, \mathcal{H}\{i\}}[k]\right\}
\end{gathered}
$$

eşitlikleri elde edilmektedir.

iii. Distorsiyon güç: Distorsiyon güç, farklı frekanslardaki gerilim ve akım işaretleri sonucu meydana gelmektedir. Diğer güç bileşenlerinde olduğu gibi gelen $\left(D_{i}\right)$, yansiyan $\left(D_{r}\right)$ ve gönderilen distorsiyon güç $\left(D_{t}\right)$ olarak ayrılmakta ve aşağıdaki eşitliklerle hesaplanmaktadır. 


$$
\begin{gathered}
D_{i}=\sqrt{V_{i}^{2} I_{r}^{2}+V_{r}^{2} I_{i}^{2}}=\sqrt{V_{1}^{2} \sum_{k=2}^{\infty} I_{k}^{2}+I_{1}^{2} \sum_{k=2}^{\infty} V_{k}^{2}} \\
D_{r}=\sqrt{\sum_{k=2}^{\infty} \sum_{\ell=2, k \neq \ell}^{\infty} V_{k}^{2} I_{\ell}^{2}} \\
D_{t}=\sqrt{D_{i}^{2}+D_{r}^{2}}=\sqrt{\sum_{k=1}^{\infty} \sum_{\ell=1, k \neq \ell}^{\infty} V_{k}^{2} I_{\ell}^{2}}
\end{gathered}
$$

DWPT kullanılarak harmoniklerin etkin değerlerinin farklı yollardan elde edilmesi (Hamid, Mardiana and Kawasaki, 2002; Vatansever and Ozdemir, 2008; Vatansever ve diğ., 2009)'de yer almaktadır. Bunlardan faydalanılarak önerilen distorsiyon güç bileşenleri, dalgacık tabanlı hesaplanabilmektedir.

iv. Yansıyan gü̧̈ kayıp faktörü: Yansıyan kayıp güç faktörü

$$
\Gamma=\frac{P_{r}}{P_{i}}
$$

şeklinde tanımlanmaktadır. Bu eşitlik dalgacık paket katsayıları cinsinden düzenlenirse

$$
\Gamma=\frac{P_{r}}{P_{i}}=\frac{\sum_{m=1}^{2^{s}-1}\left\{\frac{1}{N} \sum_{k=1}^{N / 2^{s}} p_{s}^{m, v}[k] p_{s}^{m, i}[k]\right\}}{\frac{1}{N} \sum_{k=1}^{N / 2^{s}} p_{s}^{0, v}[k] p_{s}^{0, i}[k]}
$$

elde edilmektedir.

(Lu, Lin and Huang, 2000)'de tanımlanan güç bileşenleri ve bunların Fourier tabanlı olarak gerçekleştirilen hesaplamalarının yerine alternatif olarak bu çalışmada önerilen dalgacık paket dönüşümü tabanlı işlem aşamaları, Şekil 4'te özetlenmektedir.

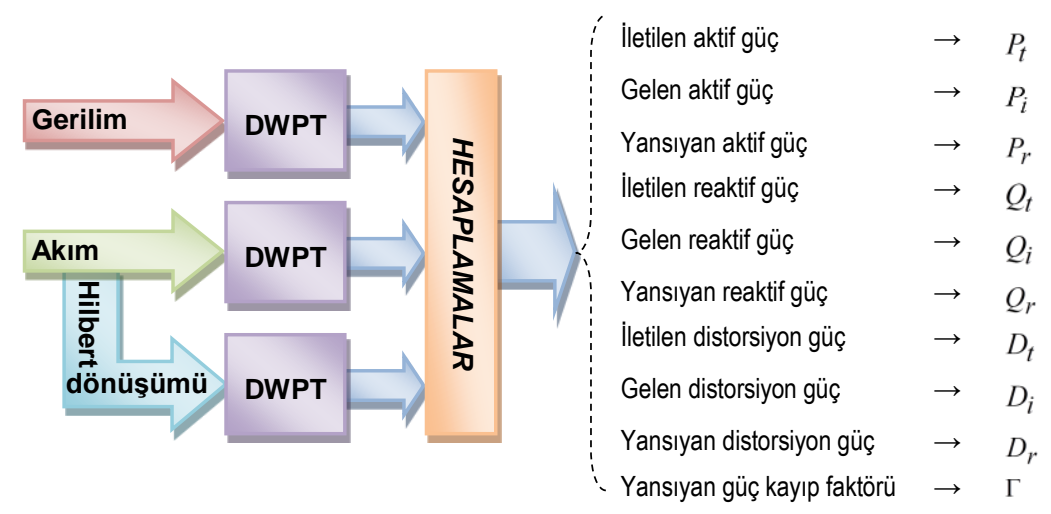

Şekil 4:

Önerilen hesaplama yönteminin blok diyagramı 


\section{BENZETIMLER}

$\mathrm{Bu}$ çalıșmada önerilen dalgacık paket dönüșümü tabanlı güç bileșenleri hesaplamalarını gerçekleştirmek için MATLAB (MathWorks, 2019) ve WaveLab (Donoho, 1999) kullanılarak etkileşimli grafiksel arayüz programı tasarlanmıştır. Kullanıcı dostu arayüze sahip ve bu türde güç analizlerinin hem gerçekleştirilmesinde hem de eğitiminde kullanılabilecek programın akış diyagramı Şekil 5 'te verilmektedir. Programın çalıştırılmasıyla oluşan ana ekran ve açıklamaları, Şekil 6'da görülmektedir. Programa gerilim ve akım verileri dışarıdan (çevre birimlerden) aktarılabildiği gibi kendi içindeki örnek veriler (gerilim ve akım dalgaları/değerleri) de kullanılabilmektedir. İşaretlerin seçilmesi veya yüklenmesi esnasında da eş zamanlı olarak grafikleri çizdirilmektedir. Analizle ilgili ayarlar (ayrı pencerede açılan ve filtre seçimi, ayrışım seviyesi belirleme gibi ayarların otomatik veya elle yapılabildiği) tamamlanıp analizin gerçekleştirilmesiyle sonuçlar görüntülenmektedir. Menü seçenekleriyle bu sonuçlar hem sayısal (gerilim ve akım işaret değerleri, analiz sonuçları) hem de grafiksel (ekran görüntüsü) olarak kaydedilebilmekte ve yazdırılabilmektedir. Ayrıca araç çubuklarıyla grafikler üzerinde büyütme, küçültme, taşıma, döndürme ve değer okuma işlemleri yapılabilmektedir. Bunun yanında "Yardım" menüsünde yer alan 'Konu anlatımı' seçeneğiyle de ilgili güç tanımlamaları ve hesaplama yöntemleriyle ilgili açıklamalara ulaşılabilmektedir.

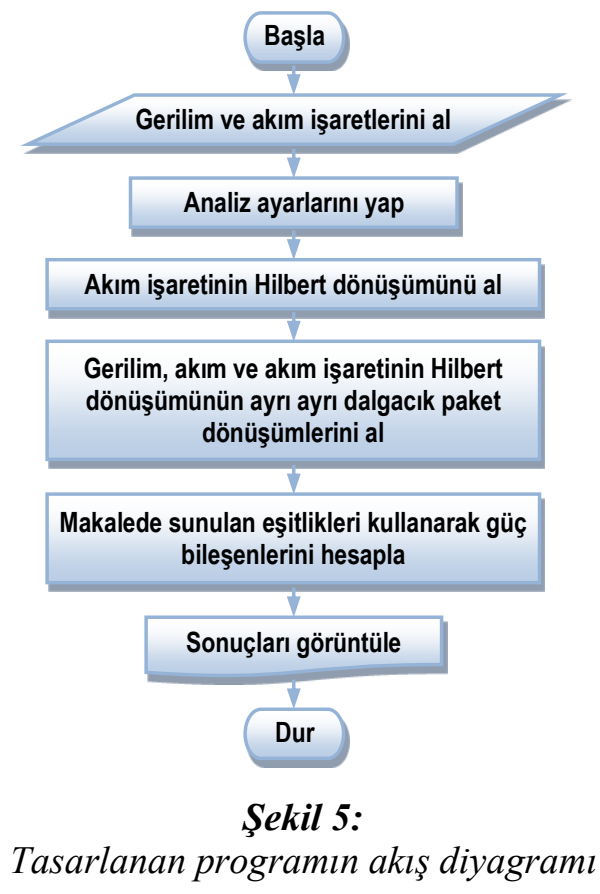

Birinci benzetim, $\omega=2 \pi 50$ olmak üzere

$$
\left.\begin{array}{l}
v(t)=\sqrt{2}\left\{220 \operatorname{Sin}(\omega t)+50 \operatorname{Sin}\left(3 \omega t+171^{\circ}\right)+20 \operatorname{Sin}\left(5 \omega t+111^{\circ}\right)\right\} \\
i(t)=\sqrt{2}\left\{44 \operatorname{Sin}\left(\omega t-15^{\circ}\right)+10 \operatorname{Sin}\left(3 \omega t+125^{\circ}\right)+4 \operatorname{Sin}\left(5 \omega t+157^{\circ}\right)\right\}
\end{array}\right\}
$$

gerilim ve akım işaretleri $6400 \mathrm{~Hz}$ ile örneklenerek 1024 değer alınmasıyla gerçekleştirilmiştir. Alınan örnek değerler, 5. seviyeye kadar Vaidyanathan dördül aynalama filtreleri (QMF) (Wickerhauser, 1994; Donoho, 1999) ile ayrışıma tabi tutulmuştur (Şekil 7). Elde edilen karşılaştırmalı sonuçlar ve bağıl hataları Tablo 1 ile Şekil 8'de verilmektedir. 
Vatansever F.: Güç Bileşenlerinin Dalgacık Dönüşümü Tabanlı Hesaplanması

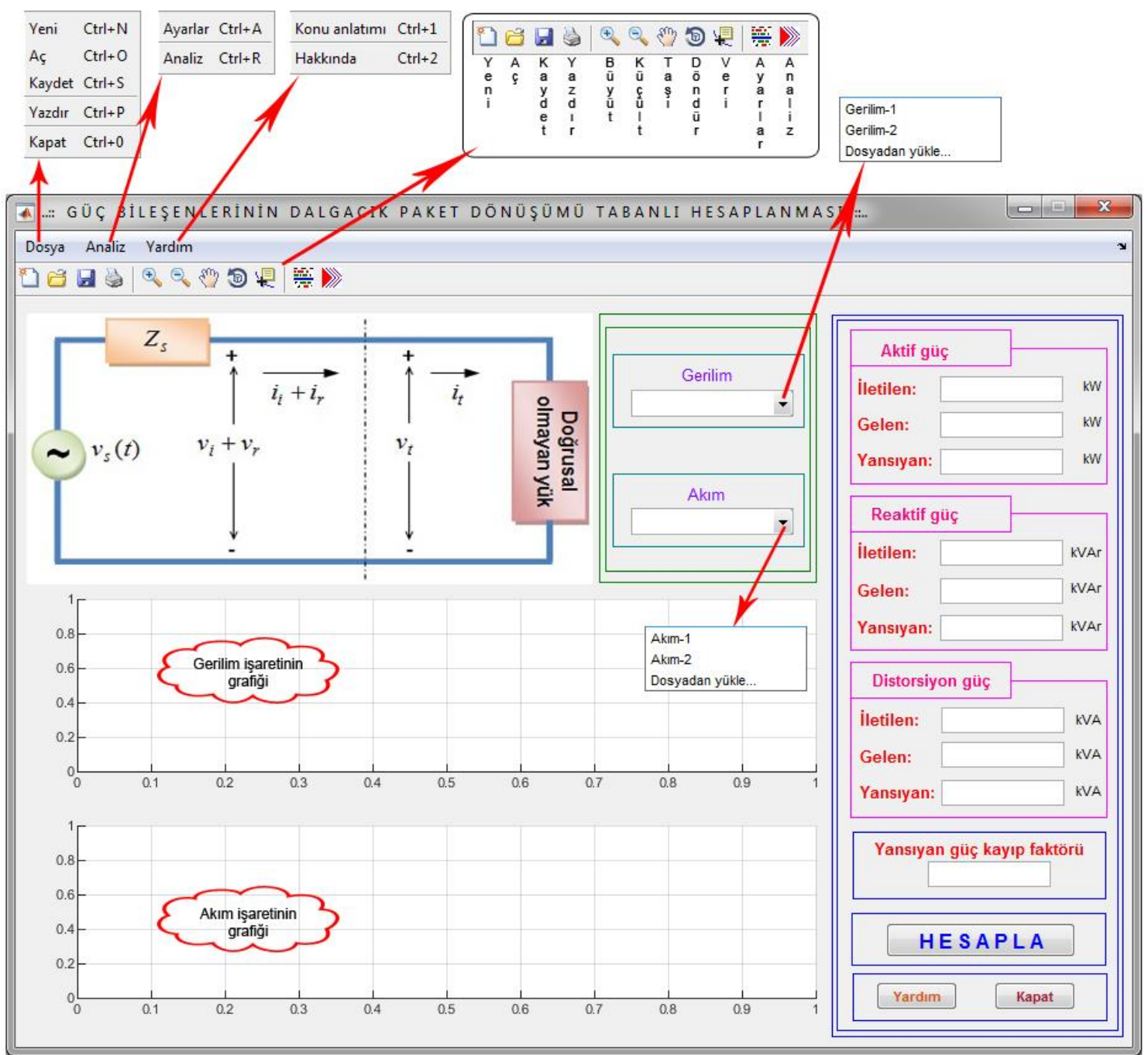

Şekil 6:

Tasarlanan programın ana ekranı ve açılklamaları

Tablo 1. Birinci benzetimin hesaplama sonuçları ve bağıl hataları

\begin{tabular}{|c|c|c|c|c|}
\hline \multicolumn{2}{|c|}{ Güç } & DWPT & Gerçek & Bağıl hata (\%) \\
\hline \multirow{3}{*}{ 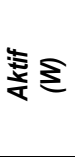 } & $P_{t}$ & 8948.0831865215495782 & 8947.2601436119621212 & 0.0091988261923409194581 \\
\hline & $P_{i}$ & 9350.5735199259124784 & 9350.1619984781809762 & 0.0044012226504576154942 \\
\hline & $P_{r}$ & -402.49033340436290018 & -402.90185486621840028 & 0.10213938131214207738 \\
\hline \multirow{3}{*}{ 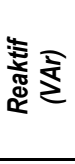 } & $Q_{t}$ & 2202.7151946918893373 & 2203.2456404501672296 & 0.024075652235014139563 \\
\hline & $Q_{i}$ & 2505.103133711573264 & 2505.3683565924006871 & 0.010586183070826270941 \\
\hline & $Q_{r}$ & -302.38793901968369937 & -302.12271614223351435 & 0.08778647327045849269 \\
\hline \multirow{3}{*}{ 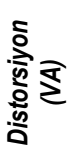 } & $D_{t}$ & 3361.8335408131183613 & 3362.8559291173924066 & 0.030402381958194061445 \\
\hline & $D_{i}$ & 3349.9386833072435365 & 3350.9401665801196941 & 0.029886635484101906068 \\
\hline & $D_{r}$ & 282.55189650363422516 & 282.84271247461900976 & 0.10281897258034941589 \\
\hline
\end{tabular}




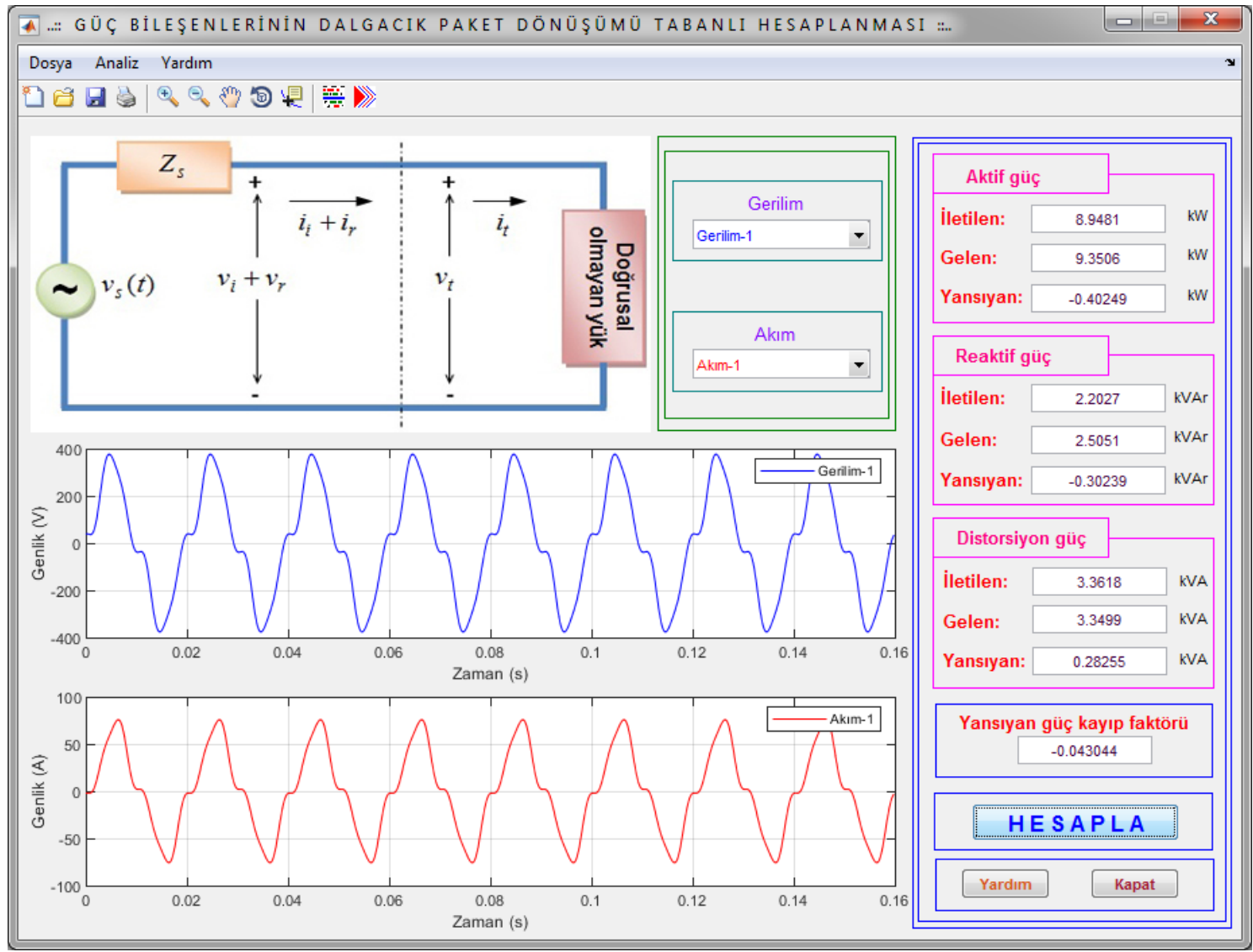

Sekil 7:

Birinci benzetimin ekran görüntüsü

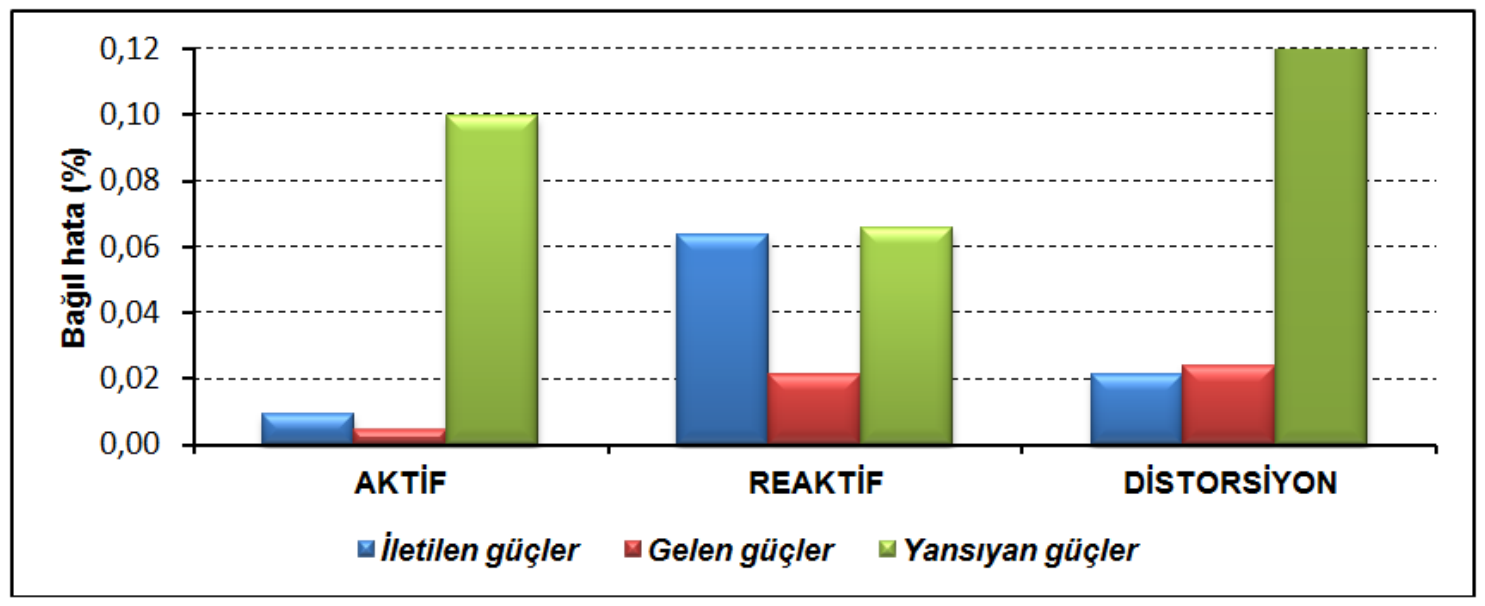

Şekil 8:

Birinci benzetimdeki bağll hata grafikleri

İkinci benzetim, $\omega=2 \pi 60$ olmak üzere

$$
\left.\begin{array}{l}
v(t)=\sqrt{2}\left\{220 \operatorname{Sin}\left(\omega t+15^{\circ}\right)+60 \operatorname{Sin}\left(3 \omega t+165^{\circ}\right)+15 \operatorname{Sin}\left(5 \omega t+110^{\circ}\right)\right\} \\
i(t)=\sqrt{2}\left\{44 \operatorname{Sin}\left(\omega t+5^{\circ}\right)+12 \operatorname{Sin}\left(3 \omega t+110^{\circ}\right)+3 \operatorname{Sin}\left(5 \omega t+175^{\circ}\right)\right\}
\end{array}\right\}
$$


gerilim ve akım işaretleri $7680 \mathrm{~Hz}$ ile örneklenerek 1024 örnek değer alınmasıyla gerçekleştirilmiştir. Alınan örnekler, 5. seviyeye kadar Vaidyanathan QMF ile ayrışıma tabi tutulmuştur (Şekil 9). Elde edilen karşılaştırmalı sonuçlar ve bağıl hatalar Tablo 2 ile Şekil 10'da verilmektedir. Tablo 1-2'de "Gerçek" olarak isimlendirilen sütundaki değerler, Eşitlik 18-19'daki gerilim ve akımlar kullanılarak doğrudan hesaplanmıştır.

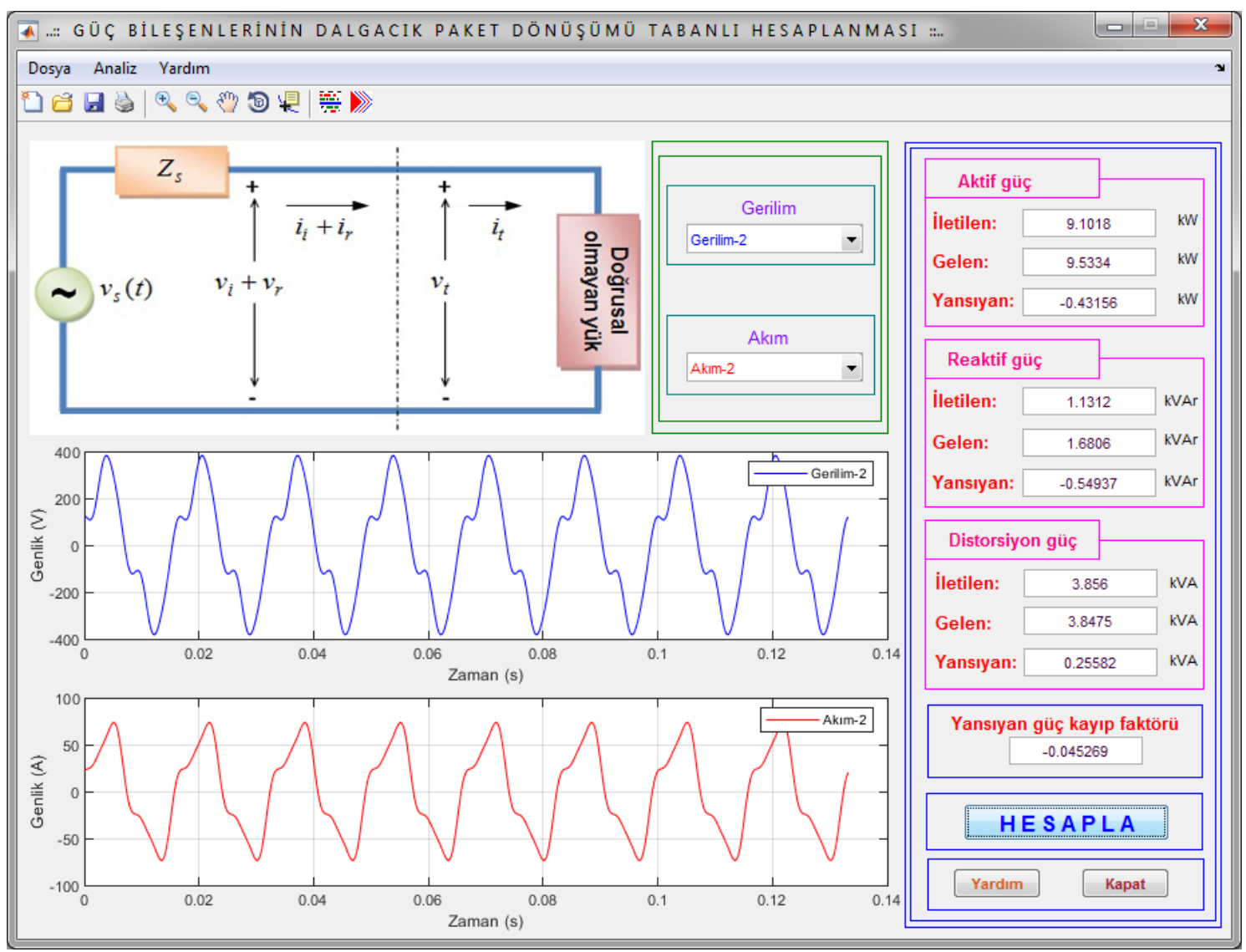

Şekil 9:

İkinci benzetimin ekran görüntüsü

Tablo 2. İkinci benzetimin hesaplama sonuçları ve bağıl hataları

\begin{tabular}{|c|c|c|c|c|}
\hline \multicolumn{2}{|c|}{ Güç } & DWPT & Gerçek & Bağıl hata (\%) \\
\hline \multirow{3}{*}{ 喜 } & $P_{t}$ & 9101.8071080985264416 & 9100.9461932070898911 & 0.0094596196171243592671 \\
\hline & $P_{i}$ & 9533.3695065966912807 & 9532.9390491581743845 & 0.0045154745697750858432 \\
\hline & $P_{r}$ & -431.56239849816563492 & -431.99285595108466396 & 0.099644576753780980671 \\
\hline \multirow{3}{*}{ 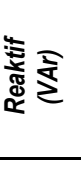 } & $Q_{t}$ & 1131.1880882184721031 & 1131.908738344460744 & 0.06366680471454524981 \\
\hline & $Q_{i}$ & 1680.5540347522082811 & 1680.9143598158855184 & 0.021436253523154178563 \\
\hline & $Q_{r}$ & -549.36594653373617803 & -549.00562147142488811 & 0.065632308344231476971 \\
\hline \multirow{3}{*}{ 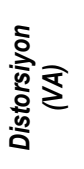 } & $D_{t}$ & 3856.0121909261438304 & 3856.8380831971671796 & 0.021413713855955210374 \\
\hline & $D_{i}$ & 3847.5168524539390091 & 3848.4282505978981135 & 0.023682347301590151584 \\
\hline & $D_{r}$ & 255.82041875888891898 & 254.55844122715710878 & 0.49575159466267182395 \\
\hline
\end{tabular}




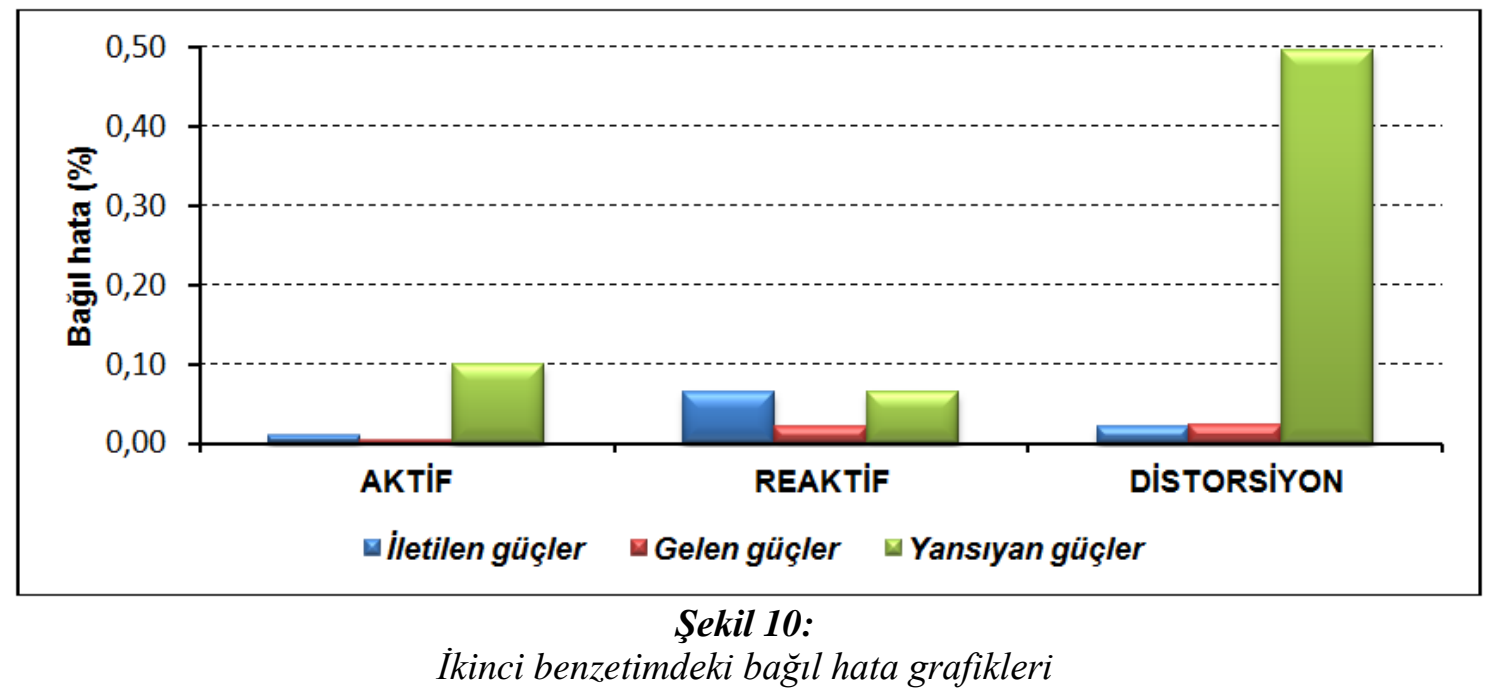

Tablo 1-2 ve Şekil 8 ile Şekil 10'daki bağıl hata oranlarından da görüldüğü gibi, Fourier tabanlı yaklaşımlara alternatif olarak DWPT kullanılarak da hesaplamalar çok yüksek doğruluklarla gerçekleştirilebilmektedir. Her iki benzetimdeki hata oranlarının çok düşük olduğu (\%0.5'ten küçük) gözlenmektedir. Gerçek değerlerle dalgacık tabanlı sonuçlar arasındaki küçük farklar, kullanılan filtrelerin doğrusal olmayan karakteristiklerinden kaynaklanmaktadır. Daha uygun filtreler tasarlanarak, özellikle harmoniklerin etkin değerlerinin hesaplanmasındaki hatalar büyük oranlarda azaltılabilecektir.

\section{SONUÇLAR}

Enerji sistemlerindeki güçler ve bunların yüksek doğruluklarla hesaplanması ve ölçülmesi son derece önemlidir. Güç analizindeki geleneksel yöntemler genellikle Fourier dönüşümlerine dayanmaktadır. Ancak sürekli ve geçici rejimlerde yapılan eş zamanlı analizlerde Fourier dönüşümlerinde problemler oluşabilmektedir. Bu nedenlerle alternatif analizler araştırılmaktadır. Gerçekleştirilen çalışmada gelen-yansıyan-iletilen aktif-reaktif-distorsiyon güç olarak tanımlanan bileşenlerin, dalgacık paket dönüşümü kullanılarak hesaplanması önerilmiştir. Hem analiz hem de eğitim amaçlı kullanılabilecek ve kullanıcı dostu arayüze sahip tasarlanan program ile yapılan benzetimlerle/uygulamalarla dalgacık paket dönüşümü tabanlı yöntem sonuçlarının, gerçek sonuçlarla örtüştüğü (çok düşük oranlarda hatalar içerdiği) gözlemlenmiştir.

\section{KAYNAKLAR}

1. Arseneau, R., Baghzouz, Y., Belanger, J., Bowes, K., Braun, A., Chiaravallo, A., Cox, M., Crampton, S., Emanuel, A., Filipski, P., Gunther, E., Girgis, A., Hartmann, D., He, S. D., Hensley, G., Iwanusiw, D., Kortebein, W., Mccomb, T., Mceachern, A., Nelson, T., Oldham, N., Piehl, D., Srinivasan, K., Stevens, R., Unruh, T., Williams, D. (1996) Practical definitions for powers in systems with nonsinusoidal waveforms and unbalanced loads: a discussion, IEEE Transastions on Power Delivery, 11 (1), 79-101. doi: https://doi.org/10.1109/61.484004

2. Budeanu, C.I. (1927) Puissances Reactives at Fictives, Institut Romain de l'Énergie, Bucharest, Romania.

3. Czarnecki, L.S. (1985) Considerations on the reactive power in nonsinusoidal situations, IEEE Transactions on Instrumentation and Measurement, 34 (3), 399-404. doi: https://doi.org/10.1109/TIM.1985.4315358 
4. Çankaya, İ., Vatansever, F. (2002) Fourier ve dalgacık dönüşümünün karşılaştırılması, SDÜ Fen Bilimleri Enstitüsü Dergisi, 6 (3), 14-24.

5. Debnath, L. (2002) Wavelet Transforms \& Their Applications, Birkhäuser, Boston.

6. Donoho, D.L. (1999) Software package 'WaveLab v.802' of MATLAB program.

7. Emanuel, A.E. (1990) Power in non-sinusoidal situations a review of definitions and physical meaning, IEEE Transactions on Power Delivery, 5 (3), 1377-1389. doi: https://doi.org/10.1109/61.57980

8. Filipski, P.S., Baghzouz, Y., Cox, M.D. (1994) Discussion of power definitions contained in the IEEE dictionary, IEEE Transactions on Power Delivery, 9 (3), 1237-1244. doi: https://doi.org/10.1109/61.311149

9. Fryze, S. (1931) Active, reactive and apparent power in non-sinusoidal systems, Przegled Elektrotek, 7, 193-203.

10. Goswami, J.C., Chan, A.K. (1999) Fundamentals of Wavelets, John Wiley\&Sons, USA.

11. Hamid, E.Y., Mardiana, R., Kawasaki, Z.I. (2002) Method for RMS and power measurements based on the wavelet packet transform, IEE Proceedings - Science, Measurement and Technology, 149 (2), 60-66. doi: https://doi.org/10.1049/ip-smt:20020156

12. IEEE (1988) IEEE Standart Dictionary of Electrical and Electronics Terms ANSI/EKE std 100-1988, IEEE, New York.

13. Kusters, N.L., Moore, W.J.M. (1980) On the definition of reactive power under nonsinusoidal conditions, IEEE Transaction on Power Apparatus and Systems, PAS-99 (5), 1845-1854. doi: https://doi.org/10.1109/TPAS.1980.319833

14. Lu, S.L., Lin, C.E., Huang, C.L. (2000) Suggested power definition and measurement due to harmonic load, Electric Power Systems Research, 53 (2), 73-81. doi: https://doi.org/10.1016/S0378-7796(98)00171-0

15. Mathworks (2019), MATLAB, www.mathworks.com

16. Sankaran, C. (2002) Power Quality, CRC Press.

17. Sharon, D. (1973) Reactive power definition and power factor improvement in non-linear systems, Proceedings of the Institution of Electrical Engineers, 120 (6), 704-706. doi: https://doi.org/10.1049/piee.1973.0155

18. Shepherd, W., Zakikhani, P. (1972) Suggested definition of reactive power for nonsinusoidal systems, Proceedings of the Institution of Electrical Engineers, 119 (9),1361-1362. doi: https://doi.org/10.1049/piee.1972.0268

19. Slonim, M.A., Van der Wyk, J.D. (1988) Power components in a system with sinusoidal and non-sinusoidal voltages and/or currents, IEE Proceedings B - Electric Power Applications, 135 (2), 76-84. doi: https://doi.org/10.1049/ip-b.1988.0010

20. Vatansever, F., Ozdemir, A. (2008) A new approach for measuring RMS value and phase angle of fundamental harmonic based on wavelet packet transform, Electric Power Systems Research, 78(1), 74-79. doi: https://doi.org/10.1016/j.epsr.2006.12.009

21. Vatansever, F., Ozdemir, A. (2009) Power parameters calculations based on wavelet packet transform, International Journal of Electrical Power and Energy Systems, 31, 596-603. doi: https://doi.org/10.1016/j.ijepes.2009.04.001 
22. Vatansever, F., Uyaroğlu, Y., Özdemir, A. (2009) Dalgacık paket tabanlı harmonik analizi, 5th International Advanced Technologies Symposium (IATS'09), Karabuk/Turkey, 13-15 May. 432-437.

23. Vatansever, F., Ozdemir, A. (2010) An alternative approach for calculating/measuring fundamental powers based on wavelet packet transform and its frequency sensitivity analysis, Electrical Engineering, 91, 417-424. doi: https://doi.org/10.1007/s00202-010-0150-x

24. Wickerhauser, M.V. (1994) Adapted Wavelet Analysis from Theory to Software, AK Peters, Wellesley.

25. Yoon, W.K., Devaney, M.J. (1998) Power measurement using the wavelet transform, IEEE Transactions on Instrumentation and Measurement, 47 (5), 1205-1210. doi: https://doi.org/10.1109/19.746584

26. Yoon, W.K., Devaney, M.J. (2000) Reactive power measurement using the wavelet transform, IEEE Transactions on Instrumentation and Measurement, 49 (2), 246-252. doi: https://doi.org/10.1109/19.843057 
